\title{
Modelo de gestión para la aplicación del control metrológico legal y la evaluación de la conformidad en equipos biomédicos
}

\author{
A.F. Ramírez Barrera ${ }^{1, \psi}$, J.F. Martínez G. ${ }^{1}$, E. Hidalgo Vásquez ${ }^{2}$ \\ ${ }^{I}$ Grupo de Investigación e Innovación Biomédica GI2B, Medellín, Colombia \\ ${ }^{2}$ Laboratorio de Metrología Biomédica ITM, Medellín, Colombia
}

Instituto Tecnológico Metropolitano - ITM

\begin{abstract}
Resumen - En la actualidad el país enfrenta grandes cambios referentes a las normas orientadas a la gestión tecnológica que se debe aplicar a los equipos biomédicos. Además de grandes cambios que hoy el Subsistema Nacional de Calidad ha presentado al control metrológico legal que se debe aplicar a algunos equipos que prestan sus servicios en el área de la salud. Dado lo anterior este trabajo pretende presentar una propuesta basada en un modelo de gestión que integra los requerimientos del control metrológico legal aplicado a los equipos biomédicos y a su vez la aplicación de procesos de medición para las actividades asociadas a la evaluación de la conformidad, utilizando como metodología un proceso de caracterización de las exigencias establecidas en las normas de Colombia asociadas al control metrológico legal y en diferentes normas para el aseguramiento de las mediciones en equipos, esto orientado a la evaluación de la conformidad, obteniendo como resultado más significativo una propuesta de estructura de gestión que le permitirá a las unidades de ingeniería de las entidades prestadoras de servicio de salud, no solo cumplir con lo exigido en las normas actuales sino a prestar unos servicios de alta calidad basados en confiabilidad como apoyo a los actividades encaminadas a la seguridad del paciente.
\end{abstract}

Palabras clave — Gestión metrológica, calibración, equipo biomédico, control metrológico legal, seguridad del paciente.

\section{Management Model for the Application of Legal Metrological Control and Conformity Assessment in Biomedical Equipment}

\footnotetext{
Abstract - At present, the country faces major changes regarding regulations oriented toward technological management to be applied to biomedical equipment. In addition to those significant changes today, the National Quality System has introduced others regarding metrological control that need to be applied to some equipment in health services. As such, this paper aims to present a proposal based on a management model which integrates legal metrological controls applied to biomedical equipment, along with the application of measurement processes for activities associated to conformity evaluation. This model also uses a method of characterization of requirements established in different regulations aimed at ensuring equipment measurements also
}

\% Dirección para correspondencia: andresramirez@itm.edu.co

DOI: https://doi.org/10.24050/19099762.n21.2017.1175 
oriented toward conformity evaluation. The most significant expected result will be the proposal of a management structure enabling engineering units of health service providers to not only comply with said regulations, but also to offer high quality services based on trustworthiness, as support for activities aimed at patient safety.

Keywords - Metrological management, calibration, biomedical equipment, legal metrological control, patient safety.

\title{
Modelo de GeStÃo PARA O APLICATIVO do CONTROLE METROLÓGiCO LEGAL E A AVALIAÇÃO DA CONFORMIDADE EM EQUIPAMENTOS BIOMÉDICOS
}

\begin{abstract}
Resumo-Na atualidade o país enfrenta grandes mudanças referentes às normas orientadas à gestão tecnológica que se deve aplicar aos equipamentos biomédicos, além de grandes mudanças que hoje o subsistema nacional de qualidade a apresentado e orientado ao controle metrológico legal que se deve aplicar a alguns equipamentos que prestam seus serviços na área da saúde, dado o anterior este trabalho pretende apresentar uma proposta baseada num modelo de gestão que integra os requerimentos do controle metrológico legal aplicado às equipamentos biomédicos e a sua vez o aplicativo de processos de medida para as atividades associadas à avaliação da conformidade, utilizando como metodologia um processo de caracterização das exigências estabelecidas nas normas da Colômbia associadas ao controle metrológico legal e ao estabelecido em diferentes normas orientadas à garantia das medidas em equipamentos, isto orientado à avaliação da conformidade, obtendo como resultado mais significativo uma proposta de estrutura de gestão que lhe permitirá às unidades de engenharia das entidades prestadoras de serviço de saúde, não só cumprir com o exigido nas normas atuais senão também a emprestar uns serviços de alta qualidade baseados em fiabilidade como apoio às atividades encaminhadas à segurança do paciente.
\end{abstract}

Palavras-chave - gestão metrológica, calibração, equipamento biomédico, controle metrológico legal, segurança do paciente.

\section{INTRODUCCIÓN}

L as entidades prestadoras del servicio de salud, entre sus líneas estratégicas establece la Gestión Tecnológica asociada a los equipos biomédicos como fundamental en sus estándares de calidad, entendiendo que la tecnología biomédica hace parte de las herramientas vitales para el desarrollo óptimo de la medicina, ofreciendo mejores resultados en las actividades de prevención, protección, tratamiento, entre otras, esto teniendo presente el buen estado de esta tecnología, desde su adquisición, pasando por su sostenimiento, hasta finalmente el proceso de baja de los mismos [1], atravesando por diferentes métodos como el aseguramiento de las mediciones durante la utilización de los equipos, como a su vez actividades que garanticen en el tiempo estas mediciones y sus resultados [2], asumiendo las recomendaciones del fabricante y apoyo de mediciones trazables, teniendo como referentes diferentes normas tanto de los equipos biomédicos como recomendaciones dados por la Organización Internacional de Metrología Legal (OIML) o por normas dependiendo de magnitudes susceptibles que controlen o midan cada uno de estos equipos.

Dado lo anterior en el país se posee diferente legislación que regula las actividades que el portador, fabricante o proveedor de tecnología médica debe tener muy claro en el momento de la utilización o comercialización de esta tecnología, como es el caso de la resolución 2003 de 2014
[3], del Ministerio de Salud y Protección Social que en su estándar de dotación, involucra actividades de mantenimiento y calibración, a su vez el decreto 4725 de 2005 [4], del mismo ministerio, definiendo que en la etapa posventa la responsabilidad del funcionamiento del equipo es compartida entre el fabricante o su representante en Colombia para el caso de los equipos importados y el propietario o tenedor, además que el titular o portador del equipo biomédico deberá garantizar, la capacidad de ofrecer servicios de soporte técnico permanente durante la vida útil del mismo, así como los repuestos y herramientas necesarias para el mantenimiento y calibración que permita conservar los equipos en los rangos de seguridad establecidos inicialmente por el fabricante.

Posteriormente el Ministerio de Comercio, Industria y Turismo, expidió el Decreto 1471 de 2014 [5] "Por el cual se reorganiza el Subsistema Nacional de Calidad y se modifica el Decreto 2269 de 1993", siendo compilado en el Decreto Único Reglamentario del Sector Comercio, Industria y Turismo del 26 de mayo de 2015 (Decreto 1074 de 2015), y finalmente modificado por el Decreto 1595 del 5 de agosto de 2015, particularmente en el Artículo 3, que modifica el Capítulo 7 del título 1 de la parte 2 del libro del decreto 1074 de 2015, estableciendo unos lineamientos claros orientados a la importancia de los procesos de medición en el país y un fortalecimiento al Subsistema Nacional de la Calidad como requisito para mejorar la competitividad y el entorno para el desarrollo 
productivo. A través del Decreto 1595 de 2015 [6], declara que están sujetos a realizar control metrológico legal específicamente los instrumentos de medida que sirven para medir, pesar y contar, y que sus actividades pueden afectar la vida, la salud o la integridad física, lo que permite inferir que los equipos biomédicos deben clasificarse en tres grandes grupos; el primer grupo sería los equipos que pesan, miden o cuentan, por lo que se les debe realizar un control metrológico legal, el segundo grupo sería los que no pesan, miden o cuentan, pero tienen subsistemas que si lo realizan, y finalmente el tercer grupo sería el de los equipos biomédicos que ni pesan, ni miden, ni cuenten, ni poseen subsistemas que lo hagan; a los dos últimos grupos anteriores si bien no estarán enmarcados en lo declarado en el Decreto 1595 del 2015, es importante realizarle unas evaluaciones de su conformidad según recomendaciones de fabricante y homologaciones de tecnología que posibiliten garantizar la confiabilidad de las mediciones o resultados encontrados. Por consiguiente, en este trabajo se presenta una propuesta de un modelo de gestión para la aplicación del control metrológico legal, según las disposiciones normativas actuales, adicionalmente presentando un conjunto de actividades integrales que permitan apoyar a la entidad prestadora del servicio de salud realizar actividades orientadas a la evaluación de la conformidad en Equipos Biomédicos.

\section{MetodologíA}

Para la realización de la propuesta del modelo de gestión para la aplicación del control metrológico legal y la evaluación de la conformidad en equipos biomédicos se tomó como base lo establecido en la legislación colombiana actual [3] [4] [5] [6], esto primero para tener claro lo relacionado a lo que aplica de control metrológico legal a los equipos biomédicos, $\mathrm{y}$ a cuales según recomendaciones de fabricante a los otros equipos, pero sin alejarnos de las exigencias que debe tener cualquier equipo de medición o que contenga un subsistema de medición el cual es crítico para la toma de decisiones, por lo tanto se tomó también como referentes lo establecido en la NTC-ISO 10012:2003 Sistema de gestión de la medición. Requisitos para los procesos de medición y los equipos de medición [7], lo establecido en la Guía Técnica Colombiana GTC63 Principios de aseguramiento del control metrológico [8], y la Guía Técnica Colombiana GTC62 Seguridad de funcionamiento y calidad de servicio. Mantenimiento. Terminología [9], todo lo anterior para dar un concepto integral de lo que requieren como mínimo la tecnología biomédica en sus procesos de medición para brindar esa confiablidad, dado que estas normas nacionales e internacionales plantean modelos específicos para asegurar las mediciones de los instrumentos, actividades que aplican completamente a los equipos biomédicos, $\mathrm{y}$ aportan en la estructura de modelos de evaluación de la conformidad orientados a la seguridad del paciente. Además, se tiene en cuenta el concepto de la Gestión del Riesgo, como actividad de identificación de los riesgos asociados a los equipos biomédicos teniendo como basa la norma ISO 31000 [10] y la NTC 5254 [11].

La propuesta plantea tres grandes procesos, (1) Evaluación de Tecnología Biomédica, (2) Fases del Control Metrológico Legal y Finalmente (3) Soporte Técnico del Equipo Biomédico, las cuales inmersamente poseen unas actividades con ciertos condicionantes que permiten analizar de manera lógica el desarrollo del aseguramiento de las mediciones aplicado a los equipos biomédicos, teniendo presente que estos condicionantes son determinados por la legislación colombiana actual, y algunas por recomendación de normas orientadas al aseguramiento de instrumentos de medición. Estos procesos no son excluyentes entre sí, al contrario, los tres procesos son armonizados por actividades que inician desde la adquisición de los equipos hasta el rechazo de los mismos, el valor agregado radica en las actividades que posee internamente cada proceso para apoyar las decisiones, las cuales se conviertan más objetivas basadas en los procesos de medición y conformidad de los equipos, dado que estas decisiones son soportadas por los datos obtenidos en las mediciones teniendo como base los resultados de error e incertidumbre.

Todo el modelo de gestión contempla el aseguramiento de las mediciones, iniciando con la adquisición de los equipos médicos la cual constituye dentro de las entidades prestadoras del servicio de salud uno de los procesos más complejos en cuanto a gestión, ya que se deben tener en cuenta diversos aspectos para obtener el resultado final que es el mejoramiento de la eficiencia y la calidad en la prestación de los servicios de salud, teniendo como punto de partida las necesidades de la parte asistencial [12]. Posterior a la adquisición de equipos basada en las necesidades de la parte asistencial, pasa a una evaluación donde se realiza la clasificación de la tecnología biomédica para determinar si requiere control metrológico legal o no, y en todo caso realizarle un soporte técnico que contemple entre sus actividades la evaluación de la conformidad determinando entre otras actividades seguridad eléctrica, gestión del riesgo y rendimiento financiero, para que finalmente se tomen decisiones objetivas y poder rechazar el equipo que no cumpla con estas condiciones e iniciar el proceso con la adquisición de un nuevo equipo biomédico si es del caso. Dado lo anterior el modelo de gestión contempla en su propuesta de evaluación de conformidad los resultados de la medición, en la toma de decisiones con los resultados de error e incertidumbre, además de un ejercicio financiero que contemple todas las variables de análisis administrativo que no esté en contravía de garantizar la 
seguridad del paciente, y finalmente estando en un marco de identificación de los riesgos asociados a los equipos biomédicos, todo en una matriz de riesgos.

\section{RESULTADOS}

El modelo de gestión para el control metrológico legal y la evaluación de la conformidad en equipos biomédicos (EB), se plantea conforme a lo establecido en diferentes conceptos de sistemas de gestión establecidos por la ISO, específicamente lo que nos plantea en los nuevos cambios de sistemas basados en procesos en la versión NTC-ISO 9001:2015, por lo que este modelo inicia con un proceso identificando las necesidades de la parte asistencial y otras partes interesadas relevantes, como lo son proveedores de tecnología médica, o proveedores de servicios especializados, esto entendiéndolo como los requisitos de entrada, para que posterior a la identificación de estas necesidades poder ejecutar lo establecido en los tres grandes procesos, (1) Evaluación de Tecnología Biomédica, (2) Fases del Control Metrológico Legal y Finalmente (3) Soporte Técnico del Equipo Biomédico, los cuales poseen actividades cíclicas y no son procesos aislados entre sí, sino que mantienen una interacción permanente, para finalmente obtener una satisfacción del cliente, bien sea interno o externo, todo en el marco de actividades orientadas a la seguridad del paciente, tal como se puede observar en la Fig1.Procediendo a la aplicación del modelo propuesto, el mismo inicia con un proceso de Evaluación de Tecnología Biomédica, tal como se evidencia en la Fig 2, donde se fundamenta en la necesidad de la parte asistencial y si esta requiere la adquisición de nuevo equipo biomédico, se analiza si el mismo es un equipo biomédico que mida, peso o cuente, dado que según lo establecido en el decreto 1595 de 2015 [6], se le debe realizar un control metrológico legal, y si no es un equipo que pese, mide o cuente, se debe analizar si al equipo biomédico (EB) el fabricante le recomienda realizarle calibración, porque si se le recomienda se le debería aplicar las mismas condiciones del control metrológico legal.

Ahora bien, si la necesidad no requiere de la compra de un equipo biomédico nuevo, entonces se les debe realizar actividades de seguimiento que si no es del grupo de los equipos biomédicos que miden pesan o cuenten, pasaría directamente al proceso de soporte técnico.

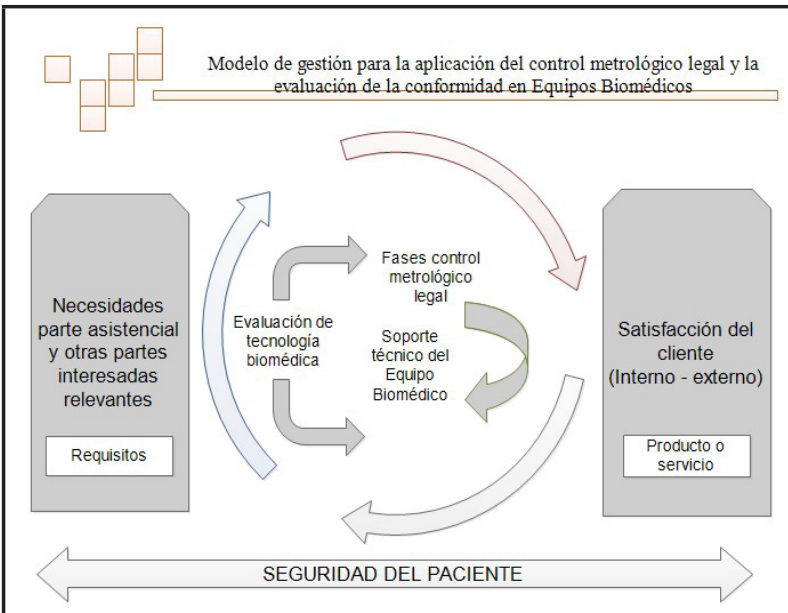

Fig. 1. Modelo de gestión para la aplicación del control metrológico legal y a evaluación de la conformidad en EB

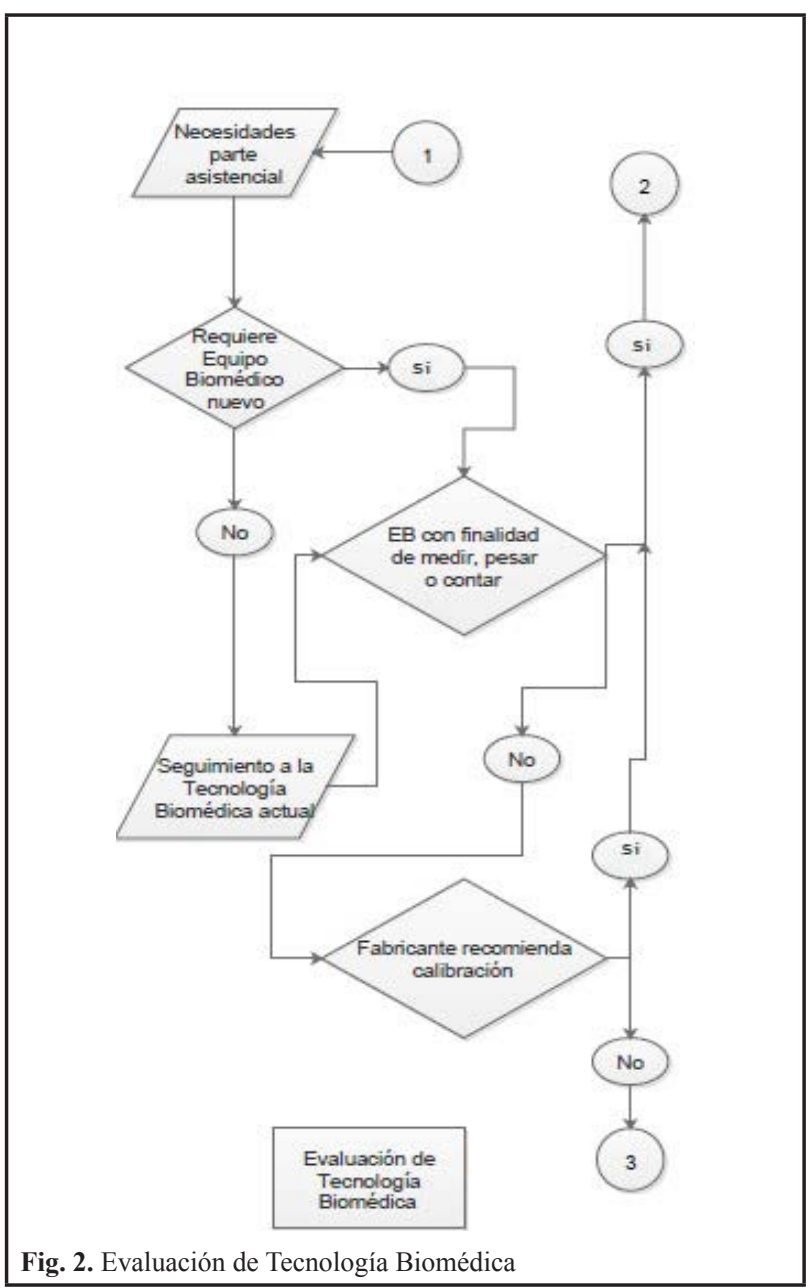

Cuando un equipo biomédico requiere de un control metrológico legal, se debe empezar con una evaluación de la conformidad tal como se observa en la Fig 3, teniendo en cuenta que esta evaluación se basa en recomendacio- 
nes del fabricante y en procesos normativos, y que es fundamental la realización de un Plan de Aseguramiento Metrológico (PAME) que posibilite hacer referencia al programa de actividades de Metrología que tiene contemplado la entidad prestadora del servicio de salud para asegurar la confiabilidad de los equipos de medición en todos y cada uno de los procesos, desde los más críticos, hasta los que solamente verifican ciertos criterios de funcionamiento, definiendo periodicidad en la calibración y los Requisitos Metrológicos del Cliente (RMC).

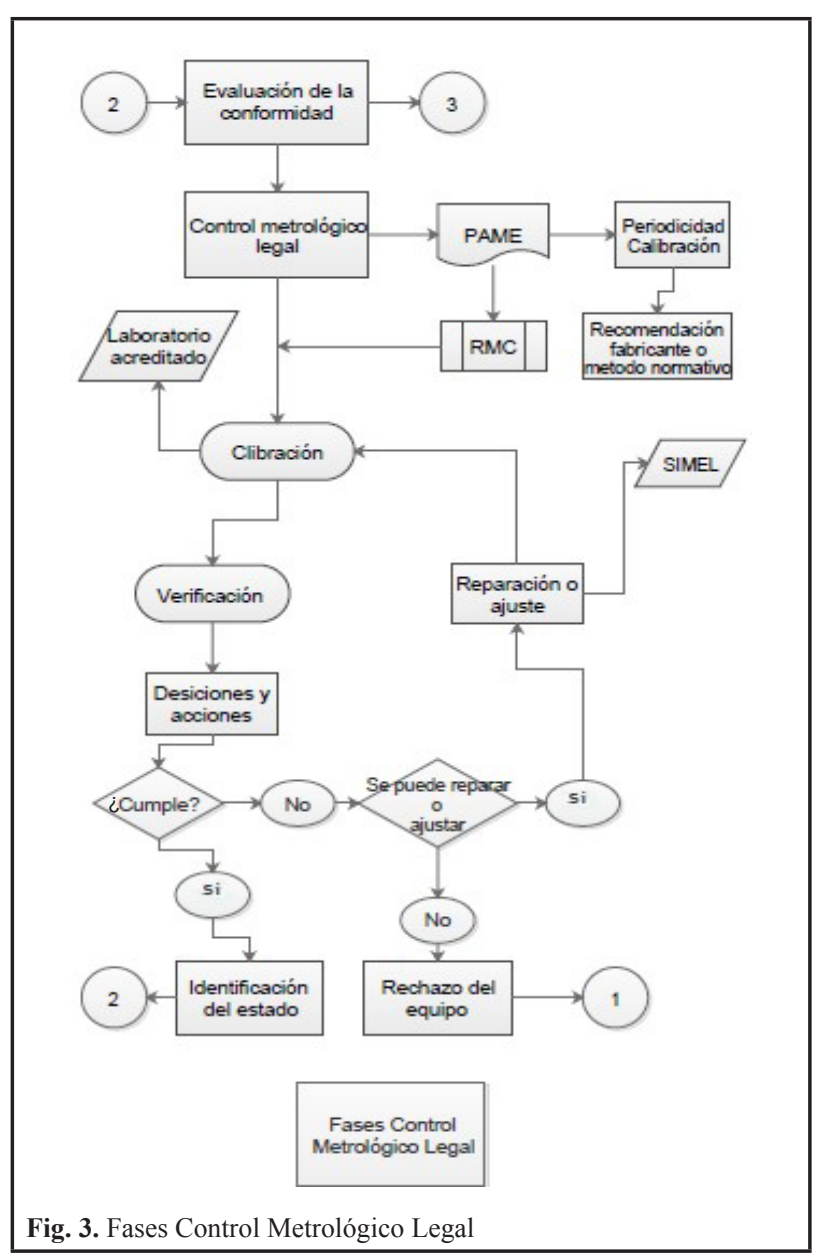

Posteriormente se realiza el proceso de calibración en laboratorios acreditados y la verificación para que consecutivamente se tomen acciones y decisiones frente a los equipos y los parámetros establecidos definiendo, si el equipo cumple, se identifica y sigue en el proceso de evaluación de la conformidad y pasa igualmente al proceso de soporte técnico para el control del mantenimiento, pero si el equipo no cumple se debe realizar una inspección para definir si el equipo se puede reparar o ajustar y en el caso que cumpla se debe proceder a realizar la reparación bien sea de forma interna, con los fabricantes o con los representantes de la marca, los cuales deben estar registrados en el Sistema de Información de Metrología Legal - SIMEL el cual integra toda la información sobre Metrología Legal de los actores que intervienen en el Control Metrológico del país, entre ellos tenemos: productores o importadores, comercializadores, reparadores y usuarios de los instrumentos de medición, de igual forma, los Organismos Autorizados de Verificación Metrológica (OAVM) y la administración como garante de la seguridad en las transacciones realizadas con dichos instrumentos.

Si posterior al proceso de reparación el equipo cumple, se procede posteriormente a realizar las actividades de calibración y verificación para garantizar que el mismo se encuentra conforme, pero en el caso que el equipo no cumpla con las especificaciones, se rechaza el equipo y pasa al proceso de evaluación de la tecnología de equipos biomédicos para analizar la necesidad y proceder a realizar la adquisición de un nuevo equipo. Finalmente el modelo de gestión para la aplicación del control metrológico legal y la evaluación de la conformidad en Equipos Biomédicos que contempla un sistema integrado de aseguramiento de las mediciones posee el proceso de soporte técnico, tal como se evidencia en la Fig 4., el cual es un proceso transversal que se aplica tanto para los equipos que requieren control metrológico legal como los que no, por lo tanto clasifica estos equipos para que los que nos les aplique el control metrológico legal, se le realice una determinación de las actividades de medición definiendo los Errores Máximos Permitidos (EMP) recomendados por los fabricantes o por normas, garantizando que estas mediciones se realicen con patrones trazables.

Consecutivamente de determinar las actividades relacionadas con los procesos de medición, pasa a ingresar los equipos biomédicos a un plan de mantenimiento preventivo como estrategia de seguimiento estricto del funcionamiento técnico de los equipos, adicionalmente se realiza un análisis del rendimiento financiero que tiene cada equipo identificando todos los costos directos e indirectos asociados a la conformidad técnica del equipo, acompañado de la realización de ensayos de seguridad eléctrica y una debida gestión del riesgo.

Si posterior a este análisis el Equipo Biomédico no cumple, se debe continuar con las actividades de reparación o ajuste correspondientes para que el equipo se encuentre en las condiciones óptimas de funcionamiento, y posteriormente ingresar nuevamente al proceso de mantenimiento preventivo; pero si en definitiva el equipo no tuvo reparación, el mismo se debe rechazar dándole de baja y pasar al proceso de evaluación de la tecnología de equipos biomédicos para analizar la necesidad y proceder a realizar la adquisición de un nuevo equipo. Finalmente tal como se puede observar en la Fig 5., los procesos inmersos en el modelo de gestión que contemplan sistema integrado 
de aseguramiento de la mediciones en equipos biomédicos es una propuesta integral de un conjunto de actividades orientadas no solo a realizar una serie de actividades que cumplan con las exigencias normativas, si no unas actividades que aportan a la confiabilidad de las mediciones y los datos suministrados por estos equipos, además que las fases propuestas en este sistema son articuladas entre si permitiendo un aporte significativo a la gestión tecnológica de las entidades prestadoras del servicio de salud.

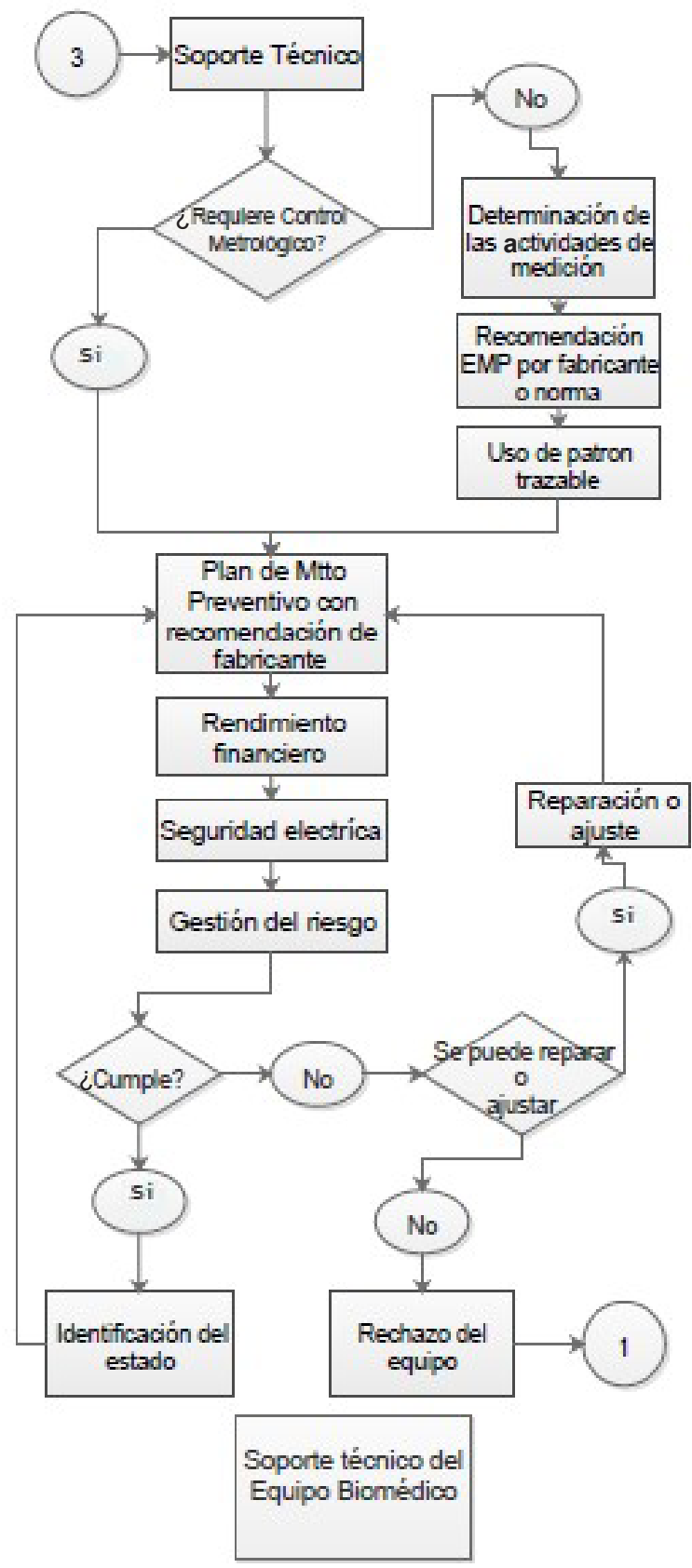

Fig. 4. Soporte Técnico del Equipo Biomédico

\section{Discusión}

Actualmente Colombia viene planteando diferentes discusiones relacionadas a la importancia o no de vincular actividades de calibración de equipos médicos en los casos en los que no aplica control metrológico legal, teniendo presente que estas actividades se encuentran inmersas los requisitos mínimos de calidad como es la habilitación, según recomendaciones del fabricante, y la inclusión de los procesos de medición en la salud con un control metrológico legal en el nuevo Subsistema Nacional de la Calidad. En este sentido, el aseguramiento de las mediciones en los equipos biomédicos es de gran importancia para los procesos de calibración y ajuste de los equipos, e indirectamente de carácter fundamental para la confiabilidad de los resultados asociados a los diagnósticos, en virtud de garantizar la seguridad del paciente en las entidades prestadoras del servicio de salud.

Tomando en cuenta la legislación colombiana, en particular, el Decreto 1595 del 2015 del Ministerio de Comercio, Industria y Turismo, la Resolución 2003 del 2014 por el Ministerio de Salud y Protección Social, y el Decreto 4725 del 2005 del mismo ministerio, se podría entregar a los pacientes una mejor calidad en el servicio, dada la certeza que los equipos médicos pueden ofrecer al estar operando bajo los estándares de calidad establecidos y la confiabilidad que agregan los resultados obtenidos de una calibración rutinaria y de aplicación de proceso de medición, mantenimiento preventivo, análisis de rendimiento financiero, seguridad eléctrica y gestión del riesgo. Así las cosas, en este trabajo presenta un modelo de gestión que contempla diferentes actividades no solo de procesos de aplicación de aseguramiento de las mediciones, si no de actividades complementarias que ayudarían a determinar una evaluación de la conformidad de los equipos biomédicos, resaltando la necesidad de promover nuevas investigaciones basadas en la legislación actual de Colombia en esta materia, de manera que se impulse la creación de nuevas metodologías, experimentos, ensayos y dispositivos, que se orienten a la mejora de la prestación de los servicios de salud.

\section{Conclusión}

Este trabajo presenta un enfoque desde la legislación vigente orientado hacia la importancia que tiene el aseguramiento de las mediciones en los equipos biomédicos, y como este impacta en las actividades orientadas a la confiablidad de los resultados, por lo tanto se presenta una propuesta integral que le permitirá a las entidades prestadoras del servicio de salud implementar estrategias de aseguramiento basadas en normatividad internacional y orientada al cumplimento de la legislación actual. 


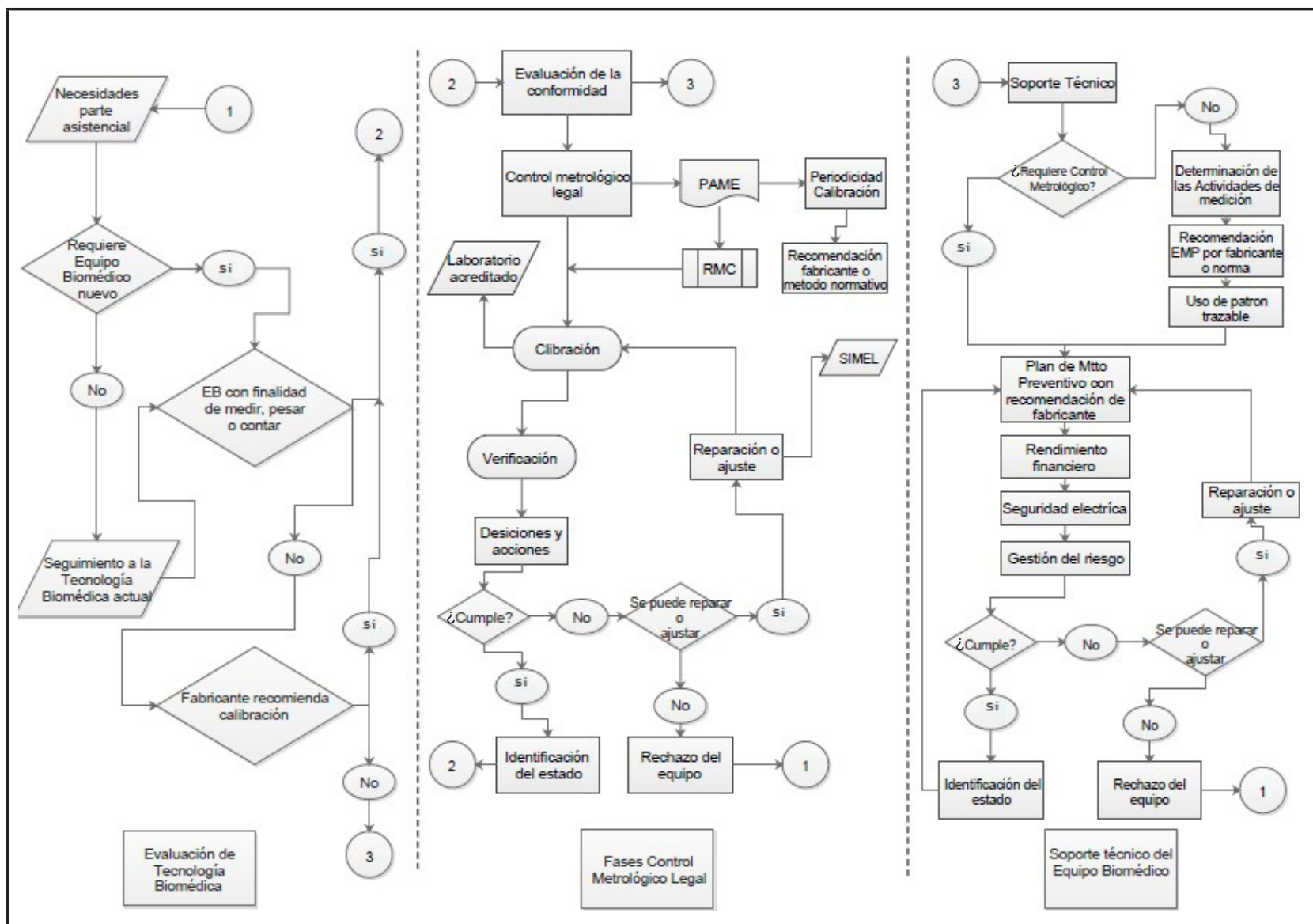

Fig. 5. Sistema Integrado de Aseguramiento de las Mediciones en Equipos Biomédicos

De otra parte, es importante resaltar que la propuesta presentada en el presente trabajo da unos puntos de partida orientada a que no solo se deben considerar los procesos de medición en los equipos biomédicos sujetos a control metrológico legal, si no que estos procesos de medición en los equipos que no pesan, miden y cuenten se debe realizar rutinariamente dado que permiten tomar decisiones bajo evidencias objetivas puesto que se deben comparar sus errores máximos permitidos con relación a los datos obtenidos, $\mathrm{y}$ esto basado en las recomendaciones de los fabricantes y recomendaciones de normas nacionales o internacionales.

\section{REFERENCIAS}

[1]. S. Grimes, The Future of Clinical Engineering: The Challenge of Change., IEEE Engineering in medicine and biology magazine, marzo-abril, 2003.

[2]. J. F. M. G. y. G. N. A. F. Ramírez Barrera, «Caracterización de la Gestión Metrológica en entidades prestadoras del servicio de salud,» Revista Ingeniería Biomédica,, vol. 9, nº 18, pp. 57-64, 2015.
[3]. MINSALUD, Resolución 2003. por la cual se definen los procedimientos y condiciones de inscripción de los Prestadores de Servicios de Salud y de habilitación de servicios de salud, Bogotá DC, 2014.

[4]. MINSALUD, Decreto 4725. Por el cual se reglamenta el régimen de registros sanitarios, permiso de comercialización y vigilancia sanitaria de los dispositivos médicos para uso humano, Bogotá DC, 2005.

[5]. MCIyT, Decreto 1471. Por el cual se reorganiza el Subsistema Nacional de la Calidad y se modifica el Decreto 2269 de 1993, Bogotá DC, 2014

[6]. MCIyT, Decreto 1595. Por el cual se dictan normas relativas al Subsistema Nacional de la Calidad y se modifica el capítulo 7 y la sección 1 del capítulo 8, Bogotá DC, 2015

[7]. ICONTEC, NTC-ISO 10012. Sistema de gestión de la medición. requisitos para los procesos de medición y los equipos de medición, 2003.

[8]. ICONTEC, GTC63 Principios de aseguramiento del control metrológico, 1999.

[9]. ICONTEC, GTC62. Seguridad de funcionamiento y calidad de servicio. Mantenimiento. Terminología., Bogotá DC: ICONTEC, 1999. 
[10]. ISO 31000:2009. Gestión de Riesgos. Principios y directrices, ISO, 2009

[11]. ICONTEC, NTC 5254. Gestión del riesgo., Bogotá DC: ICONTEC, 2006.

[12]. J. Bronzino, Clinical Engineering: Evolution of a discipline, Clinical Engineering Handbook. Elsevier, 2004 